\title{
The main determinants of intradialysis hypertension during dialysis in chronic hemodialysis patients; a single-center study
}

\author{
Mohammadreza Abbasi ${ }^{1}$, Bahareh Hajisalimi ${ }^{2 *}$ \\ ${ }^{1}$ Department of Intimal Medicine, Tehran University of Medical Sciences, Tehran, Iran \\ ${ }^{2}$ Zanjan Metabolic Diseases Research Center, Zanjan University of Medical Sciences, Zanjan, Iran
}

\section{A R T I C L E I N F O}

Article Type:

Original

\section{Article History:}

Received: 26 January 2018

Accepted: 20 April 2018

Published online: 10 May 2018

\section{Keywords:}

Intradialytic hypertension

Main determinants

Chronic hemodialysis patients

\begin{abstract}
A B S T R A C T
Introduction: Raising or lowering blood pressure and muscle cramps are the main barriers for dialysis. Meanwhile, lowering blood pressure is more common and its mechanism is somewhat clear. But the mechanism for increasing blood pressure is not yet clear fully. Objectives: In this study, we examined the prevalence of intradialytic hypertension (IDH) and its related factors, especially changes in plasma renin activity, hematocrit, heart rate, and electrolytes in end-stage renal disease (ESRD) patients undergoing chronic hemodialysis. Patients and Methods: In a cross-sectional study, patients with chronic end-stage renal disease under chronic hemodialysis were included to the study in the absence of a specific infection, as well as in lack of intravenous fluid providing during dialysis due to hypotension. Blood pressure and heart rate before and after hemodialysis were measured in four consecutive dialysis sessions. Elevating mean arterial blood pressure by $15 \mathrm{~mm} \mathrm{Hg}$ between beginning and end of dialysis or raising blood pressure within four consecutive dialysis sessions were considered as IDH.

Results: Around 17 of the 88 (19.3\%) patients with hemodialysis had IDH. These subjects were compared with non-IDH patients who were similar in age and gender. The most common underlying disease in both groups was diabetes mellitus followed by hypertension. Comparison of measured parameters before and after dialysis showed that serum potassium level was significantly decreased in both groups. Serum sodium level increased in both groups, but this increase was significant only in the control group. There were no significant changes in renin plasma activity, heart rate and hematocrit levels in both groups.

Conclusion: In this study, plasma renin activity in IDH group increased after dialysis compared to pre-dialysis. This finding requires to more test with larger sample size.
\end{abstract}

Implication for health policy/practice/research/medical education:

In this study on a group of hemodialysis patients, we found that plasma renin activity in the intradialytic hypertension group increased during dialysis and after dialysis compared to pre-dialysis, while in the control group it decreased after dialysis compared to pre-dialysis.

Please cite this paper as: Abbasi M, Hajisalimi B. The main determinants of intradialysis hypertension during dialysis in chronic hemodialysis patients; a single-center study. J Renal Inj Prev. 2018;7(3):152-159. doi: 10.15171/jrip.2018.38.

\section{Introduction}

Acute complications such as lowering blood pressure, hypertension and muscle cramps may impede easily dialysis procedure in uremic patients. Meanwhile, lowering blood pressure is more common and its mechanism is clear. However the mechanism related to raising blood pressure remains uncertain (1). There is no single definition of intradialytic hypertension (IDH) and a few criteria for this diagnosis are briefly presented. Amerling et al mentioned the IDH definition of an increase of 15 $\mathrm{mm} \mathrm{Hg}$ from the mean blood pressure between the onset and end of dialysis (2). Some researchers have argued that increased blood pressure in dialysis can be a constant increase in blood pressure (BP) during dialysis or at the end of dialysis or when dialysis begins and thus believed that the definition is not necessary to limit to numbers 
and ranges. In other words, repeating the phenomenon during dialysis sessions should be a warning and requires a clinical decision. The patient may have normal blood pressure during dialysis, but during the hemodialysis (HD) session, the patient's blood pressure may raise during and at the end of the session. This may also occur in people who have high blood pressure before dialysis and during dialysis (at checkout every hour) and when it finishes. This increase in blood pressure may be severe and accompanied by hypertension crisis. Intradialytic hypertension not only occurs in patients who initiate dialysis treatment (incidence) but also occurs in patients who are dialyzed for months or years (prevalence). Intradialytic hypertension is reported in about $10 \%$ of patients with hemodialysis. Amerling et al also reported an overall prevalence $8 \%$ for IDH in hemodialysis patients (2-4). In the study of Dorhout, this phenomenon has been reported in $5 \%$ to $15 \%$ of patients (5). Additionally, Inrig et al found that $12.2 \%$ of the patients suffered this problem (6).

Hypertension is associated with long-term and shortterm complications during dialysis. Numerous studies evaluated the importance of blood pressure have actually considered systolic and diastolic blood pressure before and after dialysis. In a study by Zager et al (7) on patients with systolic blood pressure $180 \mathrm{~mm} \mathrm{Hg}$ or diastolic blood pressure $90 \mathrm{~mm} \mathrm{Hg}$ after dialysis, the risk of cardiovascular morbidity and mortality increased by 1.96 and 1.73 times and the survival of patients was shown to be affected by IDH (7). If hypertension after dialysis is applied as a predictor of hypertension during dialysis, these data can indicate that hypertension during dialysis may be associated with adverse consequences. In this regard, an increase of $10 \mathrm{~mm} \mathrm{Hg}$ of blood pressure during dialysis in dialysis patients can be associated with an increase in the risk of hospitalization and death $(8,9)$. In patients with an increase of only $10 \mathrm{~mm} \mathrm{Hg}$ in blood pressure and systolic blood pressure by more than $120 \mathrm{~mm} \mathrm{Hg}$ in pre-dialysis period, increase the risk of death is expected. The increase in blood pressure during dialysis is due to overactive renin-angiotensin system (RAS) and sympathetic nervous system (SNS) in response to rapid ultrafiltration. The excessive activation of SNS and RAS reflexes results in a rapid or severe reduction in intravenous and cardiac preload leading load activation and cardiopulmonary reflux, which causes central sympathetic stimulation (10-12). This theory is still in doubt, but pathogenesis is believed to be more complicated might be related to the volume of extracellular fluid, peripheral vascular resistance, and the activation of sympathetic nervous system and renin-angiotensin system.

\section{Objectives}

In Iran, there is no published clinical report on the incidence and prevalence of hypertension during dialysis and the relationship between hypertension during dialysis and other variables. The aim of this study was to determine the prevalence of hypertension during dialysis and its effective factors in chronic renal failure patients undergoing chronic hemodialysis treatment to achieve early diagnosis of hypertension during dialysis, to identify its risk factors, and to prevent its complications in patients undergoing chronic hemodialysis.

\section{Patients and Methods \\ Study design}

In a prospective cohort study, patients with end-stage renal disease (ESRD) undergoing hemodialysis (dialysis three times a week and 4 hours for each session) in the hemodialysis department of Imam Khomeini hospital were studied. Patients who had a specific infection or received intravenous fluids during hemodialysis or had hypotension during dialysis were excluded from the study. Patients entered the study on the condition that they had informed consent and assured that all information was confidential and the record checklists were unnamed. Patients who had normal or elevated BP at baseline and faced with elevating mean arterial blood pressure by 15 $\mathrm{mm} \mathrm{Hg}$ between beginning and end of dialysis or had raised blood pressure within four consecutive dialysis sessions were considered as high blood pressure during dialysis.

Demographic data and medical findings related to patients including age, gender, weight, height, duration of chronic hemodialysis treatment, smoking, history of heart disease, hypertension, diabetes, hyperlipidemia, and history of used drugs such as erythropoietin and its doses were collected by an interviewing and recorded in the study checklist. The patient's blood pressure was assessed through the hand without vascular access before the onset of hemodialysis. Patient heart rate was first checked hourly and then at the end of dialysis. The venous blood sample was taken after at least 12 hours fasting for assessing the serum levels of triglyceride, total cholesterol, HDL-C, LDL-C, sodium, potassium and hematocrit just before hemodialysis. Serum levels of hematocrit and plasma renal activity, sodium and potassium were measured immediately after dialysis. Samples for each specific test were examined by a single specific kit. To measure the levels of HDL-C, LDL-C, sodium, potassium, hematocrit, triglyceride, and total cholesterol, kits provided by the Pars-Azmoon Company, Iran. The adequacy of dialysis was determined using the $\mathrm{Kt} / \mathrm{V}_{\text {urea }}$ formula, where $K$ is urea clearance in $\mathrm{ml}$ per minute, $t$ is the length of an HD session in minutes, and $V$ is the volume of distribution of urea in milliliters which is assumed to be equal to total body water (TBW).

Patients selected for study underwent regular hemodialysis using dialysis bicarbonate. Dialysis was performed on the basis of a normal Fresenius 4008 device and a lowflux polysulfone dialyzer. The dialyzer temperature was maintained at $36.5^{\circ} \mathrm{C}$. Individual ultrafiltration values (specific for each individual) were kept constant to achieve a dry weight. The cross section was $1.7 \mathrm{~m}^{2}$. The blood 
flow velocity was $200-250 \mathrm{~mL} / \mathrm{min}$ and dialysis fluid flow rate was $500 \mathrm{~mL} / \mathrm{min}$. For all patients, the steady state of sodium, magnesium, calcium and other electrolytes were used in dialysis fluid. The amount of ultrafiltration was maintained constant over time. Dry weight of patients was evaluated through clinical evaluation with test and error. In summary, dry weight was evaluated on the basis of regular examination during dialysis sessions.

\section{Ethical issues}

Study related information was explained to all patients and informed consent was obtained. The research followed the tenets of the Declaration of Helsinki. The Ethics Committee of Tehran University of Medical Sciences approved the study. This study was conducted as the nephrology fellowship of Bahareh Hajisalimi in Tehran University of Medical Sciences.

\section{Statistical analysis}

For statistical analysis, results were presented as mean \pm standard deviation (SD) for quantitative variables and were summarized by absolute frequencies and percentages for categorical variables. Normality of the data was analyzed using the Kolmogorov-Smirnov test. Categorical variables were compared using chi-square test or Fisher's exact test when more than $20 \%$ of cells with an expected count of less than 5 were observed. Quantitative variables were also compared with $t$ test or Mann-Whitney $\mathrm{U}$ test. For the statistical analysis, the statistical software SPSS version 16.0 for Windows (SPSS Inc., Chicago, IL) was used. $P$ values of 0.05 or less were considered statistically significant.

\section{Results}

Of 88 eligible patients, 17 (19.3\%) had hypertension within dialysis, which was compared with the same proportion of people who did not have hypertension. Comparison of demographic and clinical characteristics of patients in both hypertensive and normotensive groups showed no significant difference in age, weight, height, body mass index, lipid profiles, and dose of erythropoietin. The mean dose of erythropoietin in the group with hypertension during dialysis was numerically higher than the non-hypertensive group during dialysis but was not statistically significant (Table 1 ). Of patients without
Table 1. Demographic and clinicopathological characteristics in patients with and without intradialytic hypertension

\begin{tabular}{|c|c|c|c|c|c|}
\hline Factor & IDH & $\mathbf{N}$ & Mean & SD & $\boldsymbol{P}^{\mathrm{a}}$ \\
\hline \multirow{2}{*}{ Age } & - & 17 & 66.00 & 13.15 & \multirow{2}{*}{0.897} \\
\hline & + & 17 & 65.41 & 13.22 & \\
\hline \multirow{2}{*}{ Smoking (cigarette/day) } & - & 2 & 7.00 & 1.41 & \multirow{2}{*}{0.333} \\
\hline & + & 1 & 10.00 & & \\
\hline \multirow{2}{*}{ Weight (kg) } & - & 17 & 62.79 & 9.88 & \multirow{2}{*}{0.620} \\
\hline & + & 17 & 64.61 & 11.24 & \\
\hline \multirow{2}{*}{ Height (m) } & - & 17 & 165.76 & 9.65 & \multirow{2}{*}{0.485} \\
\hline & + & 17 & 163.47 & 9.28 & \\
\hline \multirow{2}{*}{ Body mass index $\left(\mathrm{kg} / \mathrm{m}^{2}\right)$} & - & 17 & 22.41 & 3.55 & \multirow{2}{*}{0.323} \\
\hline & + & 17 & 23.59 & 3.28 & \\
\hline \multirow{2}{*}{ Cholesterol (mg/dL) } & - & 17 & 181.06 & 43.76 & \multirow{2}{*}{0.518} \\
\hline & + & 17 & 170.41 & 50.92 & \\
\hline \multirow{2}{*}{ LDL-C (mg/dL) } & - & 17 & 109.41 & 26.77 & \multirow{2}{*}{0.385} \\
\hline & + & 17 & 99.41 & 38.34 & \\
\hline \multirow{2}{*}{ HDL-C (mg/dL) } & - & 17 & 49.29 & 14.89 & \multirow{2}{*}{0.189} \\
\hline & + & 17 & 42.65 & 13.95 & \\
\hline \multirow{2}{*}{ Triglyceride (mg/dL) } & - & 17 & 171.88 & 76.79 & \multirow{2}{*}{0.329} \\
\hline & + & 17 & 204.47 & 111.57 & \\
\hline \multirow{2}{*}{ FBS (mg/dL) } & - & 17 & 146.71 & 79.96 & \multirow{2}{*}{0.988} \\
\hline & + & 17 & 146.24 & 99.34 & \\
\hline \multirow{2}{*}{ Erythropoietin (U/kg/wk) } & - & 15 & 138.09 & 56.68 & \multirow{2}{*}{$0.1^{b}$} \\
\hline & + & 11 & 167.88 & 49.54 & \\
\hline
\end{tabular}

${ }^{\mathrm{a}} t$ test, ${ }^{\mathrm{b}}$ Mann-Whitney $\mathrm{U}$ test.

hypertension during dialysis, 8 (47.1\%) were male and $9(52.9 \%)$ were female. In the group with hypertension during dialysis, 8 (47.1\%) were female and 9 (52.9\%) were male. The two groups did not have a significant difference in gender $(P=0.7)$. The history of diabetes in those with and without dialysis-related hypertension was $43.8 \%$ and $31.3 \%$ respectively with no difference $(P=0.4)$. There was also no difference in the previous history of hypertension between the groups with and without raising blood pressure within dialysis $(67.5 \%$ versus $70.6 \% ; P=1.0)$. The two groups were also similar in the history of smoking $(P=1.00)$. The most common underlying disorder in both groups with and without raising blood pressure during dialysis was diabetes followed by hypertension (Table 2). Similarly, no difference in anti-hypertensive drug administration between the groups with and without raising blood pressure within dialysis was detected $(17.6 \%$ versus $29.4 \% ; P=0.6$ ). Of patients under study, 10 cases

Table 2. The type of underlying disease in groups with and without intradialytic hypertension

\begin{tabular}{|c|c|c|c|c|c|c|}
\hline \multirow{2}{*}{ Type of underlying renal diseases } & \multicolumn{2}{|c|}{ With Intradialytic hypertension } & \multicolumn{2}{|c|}{ Without intradialytic hypertension } & \multicolumn{2}{|c|}{ Total } \\
\hline & No. & $\%$ & No. & $\%$ & No. & $\%$ \\
\hline Unknown & 1 & 5.9 & 3 & 17.6 & 4 & 11.8 \\
\hline ADPKD & 1 & 5.9 & 2 & 11.7 & 3 & 8.8 \\
\hline Glomerolo nephritis & 3 & 17.6 & 1 & 5.9 & 4 & 11.8 \\
\hline Nephrolithiasis & 1 & 5.9 & 0 & 0 & 1 & 2.9 \\
\hline Diabetes mellitus & 7 & 41.2 & 6 & 35.3 & 13 & 38.2 \\
\hline Hypertension & 4 & 23.5 & 4 & 23.5 & 8 & 23.5 \\
\hline UTI & 0 & 0 & 1 & 5.9 & 1 & 2.9 \\
\hline Total & 17 & 100 & 17 & 100 & 34 & 100 \\
\hline
\end{tabular}


in the affected group and 10 in the non-affected group used angiotensin converting enzyme inhibitor (ACEI), which was not significantly different. The drug commonly used in both groups was losartan. Of patients under study, 4 patients in the affected group and 4 in the nonaffected group used beta-receptor blockers, which was not significant. Also, three patients in the hypertensive group and five individuals in the normotensive group consumed other lowering blood pressure medications while the difference between these two groups was not significant $(P=0.2)$. Another drug administered in patients was calcium channel blockers that six cases in each group used this drug (including diltiazem in a case and amlodipine in five cases of each group). As shown in Table 3, there was no difference between groups in the changes of hemodynamic parameters (blood pressure and heart rate), duration of dialysis and ultrafiltration, serum electrolytes, plasma renin activity, and hematocrit after dialysis as compared to before dialysis session. We could not also find any difference in the prevalence rate of heart diseases including ischemic heart disease or heart failure across the two groups with and without intradialytic hypertension (Table 4).

The adequacy of dialysis was equal to or greater than 1.2 in 15 affected by intradialytic hypertension and in 11 non-affected by intradialytic hypertension indicating no difference between the two groups. Regarding body mass index (BMI), 8 of the affected group and 10 of the non-affected group had a BMI of less than $25 \mathrm{~kg} / \mathrm{m}^{2}$ and 8 of the affected group and 3 of the non-affected group had a BMI of $25 \mathrm{~kg} / \mathrm{m}^{2}$ or more that was not different between the two groups $(P=0.13)$. The administration of erythropoietin did not differ significantly between the two groups with and without hypertension during dialysis $(P=0.1)$. Comparison of mean systolic and diastolic blood pressure and mean arterial pressure (MAP) in the affected and non-affected group showed that after dialysis, systolic and diastolic blood pressure and MAP increased significantly. In the control group, the mean systolic, diastolic blood pressure and MAP after the third dialysis decreased significantly (Table 5).

The mean heart rate decreased significantly after the first $(P=0.001)$, third $(P<0.001)$ and fourth $(P<0.001)$ dialysis session in the intradialytic hypertension group and adversely increased in the second dialysis session $(P=0.006)$. Plasma levels of hematocrit and renin increased significantly in intradialytic hypertension group after dialysis than before. In the group with intradialytic hypertension, the mean of sodium and potassium levels after dialysis did not change significantly as compared to before dialysis. In control group, the mean heart rate significantly lowered after the first $(P=0.008)$, second

Table 3. The levels of serum electrolytes, plasma renin activity, and hematocrit before and after dialysis in groups with and without intradialytic hypertension

\begin{tabular}{|c|c|c|c|c|c|}
\hline Serum electrolytes & Intradialytic hypertension & $\mathbf{N}$ & Mean & SD & $\boldsymbol{P}^{\text {a }}$ \\
\hline \multirow{2}{*}{ Serum $\mathrm{Na}$ before dialysis (meq/L) } & + & 17 & 137.06 & 3.68 & \multirow{2}{*}{0.779} \\
\hline & - & 17 & 136.59 & 5.77 & \\
\hline \multirow{2}{*}{ Serum $\mathrm{Na}$ after dialysis (meq/L) } & + & 17 & 139.71 & 1.86 & \multirow{2}{*}{0.464} \\
\hline & - & 17 & 139.12 & 2.69 & \\
\hline \multirow{2}{*}{ Plasma rennin activity before $(\mathrm{ng} / \mathrm{mL} / \mathrm{h})$} & + & 17 & 6.29 & 10.74 & \multirow{2}{*}{$0.2^{b}$} \\
\hline & - & 17 & 1.93 & 2.03 & \\
\hline \multirow{2}{*}{ Plasma rennin activity after dialysis $(\mathrm{ng} / \mathrm{mL} / \mathrm{h})$} & + & 17 & 6.20 & 9.97 & \multirow{2}{*}{$0.3^{b}$} \\
\hline & - & 17 & 2.37 & 2.64 & \\
\hline \multirow{2}{*}{ Hematocrit before dialysis (\%) } & + & 17 & 33.89 & 5.91 & \multirow{2}{*}{0.518} \\
\hline & - & 17 & 32.53 & 6.21 & \\
\hline \multirow{2}{*}{ Hematocrit after dialysis (\%) } & + & 17 & 34.54 & 4.96 & \multirow{2}{*}{0.794} \\
\hline & - & 17 & 34.07 & 5.29 & \\
\hline \multirow{2}{*}{ Serum $\mathrm{K}$ before dialysis (meq/L) } & + & 17 & 4.91 & 1.01 & \multirow{2}{*}{0.859} \\
\hline & - & 17 & 4.85 & 0.69 & \\
\hline \multirow{2}{*}{ Serum $\mathrm{K}$ after dialysis (meq/ $\mathrm{L}$ ) } & + & 17 & 3.60 & 0.49 & \multirow{2}{*}{0.421} \\
\hline & - & 17 & 3.75 & 0.59 & \\
\hline
\end{tabular}

${ }^{a}$ t test, ${ }^{b}$ Mann-Whitney $U$ test.

Table 4. History of heart disease and heart failure in groups with and without intradialytic hypertension

\begin{tabular}{|c|c|c|c|c|c|c|}
\hline \multirow{2}{*}{ Type of diseases } & & \multicolumn{2}{|c|}{ With intradialytic hypertension } & \multicolumn{2}{|c|}{ Without intradialytic hypertension } & \multirow{2}{*}{$P$} \\
\hline & & No. & $\%$ & No. & $\%$ & \\
\hline \multirow{2}{*}{ Herat Diseases } & + & 9 & 52.9 & 5 & 29.4 & \multirow{2}{*}{0.16} \\
\hline & - & 8 & 47.1 & 12 & 70.6 & \\
\hline \multirow{2}{*}{ Ischemic heart diseases } & + & 8 & 47.1 & 5 & 29.4 & \multirow{2}{*}{1} \\
\hline & - & 9 & 52.9 & 12 & 70.6 & \\
\hline \multirow{2}{*}{ Heart failure } & + & 1 & 5.8 & 1 & 5.8 & \multirow{2}{*}{0.1} \\
\hline & - & 16 & 94.2 & 16 & 94.2 & \\
\hline
\end{tabular}


Table 5. The mean systolic, diastolic, and mean blood pressures in groups with and without intradialytic hypertension

\begin{tabular}{|c|c|c|c|c|c|c|}
\hline \multirow{2}{*}{ Parameter } & \multicolumn{3}{|c|}{ With Intradialytic Hypertension } & \multicolumn{3}{|c|}{ Without Intradialytic Hypertension } \\
\hline & Mean & SD & $P$ & Mean & SD & $P$ \\
\hline Systolic BP before dialysis1 & 143.82 & 38.42 & \multirow{2}{*}{0.001} & 156.06 & 15.98 & \multirow[t]{2}{*}{0.476} \\
\hline Systolic BP after dialysis 1 & 141.06 & 35.13 & & 140.71 & 29.88 & \\
\hline Diastolic BP before dialysis 1 & 84.88 & 24.96 & \multirow{2}{*}{0.4} & 87.24 & 10.88 & \multirow{2}{*}{0.3} \\
\hline Diastolic BP after dialysis 1 & 81.65 & 11.62 & & 81.76 & 15.07 & \\
\hline MAP before Dialysis 1 & 104.49 & 27.03 & \multirow{2}{*}{0.06} & 110.14 & 10.79 & \multirow{2}{*}{0.5} \\
\hline MAP after Dialysis 1 & 101.43 & 18.84 & & 103.47 & 19.72 & \\
\hline Systolic BP before dialysis 2 & 142.41 & 29.36 & \multirow{2}{*}{0.008} & 152.24 & 22.18 & \multirow{2}{*}{0.3} \\
\hline Systolic BP after dialysis 2 & 141.00 & 35.89 & & 134.82 & 27.77 & \\
\hline Diastolic BP before dialysis 2 & 82.00 & 17.57 & \multirow{2}{*}{0.03} & 86.00 & 11.02 & \multirow{2}{*}{0.1} \\
\hline Diastolic BP after dialysis 2 & 85.52 & 18.26 & & 78.00 & 15.32 & \\
\hline Map before dialysis 2 & 102.10 & 19.67 & \multirow{2}{*}{0.015} & 108.04 & 12.84 & \multirow{2}{*}{0.2} \\
\hline Map after dialysis 2 & 103.98 & 22.08 & & 96.96 & 18.25 & \\
\hline Systolic BP before dialysis 3 & 136.65 & 30.21 & \multirow{2}{*}{0.02} & 147.35 & 18.57 & \multirow{2}{*}{0.048} \\
\hline Systolic BP after dialysis 3 & 137.65 & 28.75 & & 136.94 & 24.27 & \\
\hline Diastolic BP before dialysis 3 & 76.76 & 15.44 & \multirow{2}{*}{0.01} & 82.59 & 15.72 & \multirow{2}{*}{0.002} \\
\hline Diastolic BP after dialysis 3 & 77.94 & 13.47 & & 76.88 & 13.61 & \\
\hline Map before dialysis 3 & 96.90 & 19.40 & \multirow{2}{*}{0.02} & 104.14 & 15.61 & \multirow{2}{*}{0.001} \\
\hline Map after dialysis 3 & 97.72 & 16.50 & & 96.85 & 16.11 & \\
\hline Systolic BP before dialysis 4 & 133.88 & 28.55 & \multirow{2}{*}{0.004} & 147.53 & 20.03 & \multirow{2}{*}{0.13} \\
\hline Systolic BP after dialysis 4 & 140.41 & 36.25 & & 142.88 & 27.87 & \\
\hline Diastolic BP before dialysis 4 & 79.18 & 16.68 & \multirow{2}{*}{0.01} & 85.47 & 17.65 & \multirow{2}{*}{0.022} \\
\hline Diastolic BP after dialysis 4 & 84.41 & 18.67 & & 80.65 & 15.02 & \\
\hline Map before dialysis 4 & 94.25 & 19.43 & \multirow{2}{*}{0.004} & 106.57 & 16.84 & \multirow{2}{*}{0.049} \\
\hline Map after dialysis 4 & 103.04 & 22.98 & & 100.91 & 18.83 & \\
\hline
\end{tabular}

$(P=0.025)$, third $(P=0.016)$ and fourth $(P<0.001)$ sessions of dialysis. In this group, the mean of sodium and potassium after dialysis did not change compared to predialysis time ( $P=.6$ and $P=0.4$ respectively), but the mean of potassium significantly decreased $(P=0.003)$. Plasma renin activity decreased significantly in the control group after dialysis than before dialysis $(P<0.001)$. Hematocrit significantly increased in the control group after dialysis than before dialysis $(P=0.02)$ (Table 6).

\section{Discussion}

Raising blood pressure, lowering blood pressure and muscle cramps are acute complications of dialysis, which causes serious problems for such patients. Meanwhile, lowering blood pressure is more common and its mechanism is somewhat known. However the mechanism for increasing blood pressure is not yet clear (1). In our study, 88 patients under hemodialysis who had inclusion criteria were studied that 17 (19.3\%) had hypertension during dialysis. The same number of non-hypertensive patients who were similar in age and gender were selected as control group. Demographic and para-clinical characteristics of patients including age, weight, height, body mass index, profile lipid and dose of erythropoietin used in the two groups of patients with and without hypertension during dialysis were not significantly different. There was no significant difference in blood pressure before and after first, second, third and fourth dialysis sessions in both patients with and without hypertension during dialysis. Additionally, heart rate, dialysis duration and ultrafiltration of patients, serum $\mathrm{Na}$ and K electrolytes, plasma renin and hematocrit of patients was not significantly different before and after four dialysis stages in both groups of patients with and without hypertension during dialysis. The frequency of elevated blood pressure $\geq 10 \mathrm{~mm} \mathrm{Hg}$ in four dialysis stages, did not differ significantly between the two groups with and without hypertension during dialysis, but the mean increase in arterial blood pressure $\geq 15 \mathrm{~mm} \mathrm{Hg}$, at least in one dialysis stage, were significantly higher in patients with hypertension during dialysis $(P<0.001)$. The most common underlying disease in the two groups was diabetes mellitus, followed by hypertension. The mean of systolic and diastolic blood pressure increased significantly in the group suffered raised blood pressure within dialysis and also after the fourth session of dialysis, but adversely, the mean of systolic and diastolic blood pressures decreased significantly in the control group after the fourth session of dialysis.

As an important result, the frequency of IDH in our study was $19.3 \%$. Amerling et al (2) in a cohort study in dialysis patients reported IDH in $8 \%$ of patients. In the study by Dorhout (5), this phenomenon occurred in 5\% to $15 \%$ of patients. Inrig et al, in his large cohort study found IHD in $12.2 \%$ of patients (6) indicating an overall high rate of IDH in our observation.

Ultrafiltration increases the activity of renin-angiotensin system (RAS), which leads to excessive stimulation of renin and the production of angiotensin II. Patients 
Table 6. The mean heart rate, plasma renin activity and serum sodium and potassium levels in groups with and without intradialytic hypertension

\begin{tabular}{|c|c|c|c|c|c|c|}
\hline Parameter & Mean & SD & $P$ value & Mean & SD & $P$ value \\
\hline Heart rate per minute before dialysis 1 & 79.65 & 14.91 & \multirow{2}{*}{0.001} & 75.29 & 12.43 & \multirow{2}{*}{0.008} \\
\hline Heart rate per minute after dialysis 1 & 75.94 & 14.92 & & 74.71 & 12.81 & \\
\hline Heart rate per minute before dialysis 2 & 76.41 & 14.24 & \multirow{2}{*}{0.006} & 74.53 & 10.47 & \multirow{2}{*}{0.025} \\
\hline Heart rate per minute after dialysis 2 & 80.29 & 15.02 & & 72.41 & 13.82 & \\
\hline Heart rate per minute before dialysis 3 & 76.18 & 13.16 & \multirow{2}{*}{$<0.001$} & 74.82 & 10.32 & \multirow{2}{*}{0.016} \\
\hline Heart rate per minute after dialysis 3 & 75.18 & 13.31 & & 72.18 & 14.86 & \\
\hline Heart rate per minute before dialysis 4 & 82.59 & 16.42 & \multirow{2}{*}{$<0.001$} & 76.65 & 10.61 & \multirow{2}{*}{$<0.001$} \\
\hline Heart rate per minute after dialysis 4 & 81.00 & 16.03 & & 74.94 & 13.66 & \\
\hline Serum Na before dialysis (meq/L) & 136.59 & 5.77 & \multirow{2}{*}{0.7} & 137.06 & 3.68 & \multirow{2}{*}{0.6} \\
\hline Serum $\mathrm{Na}$ after dialysis(meq/L) & 139.12 & 2.69 & & 139.71 & 1.86 & \\
\hline Plasma rennin activity before (ng/mL/h) & 1.93 & 2.03 & \multirow{2}{*}{0.01} & 6.29 & 10.74 & \multirow{2}{*}{$<0.001$} \\
\hline Plasma rennin activity after dialysis (ng/mL/h) & 2.37 & 2.64 & & 6.20 & 9.97 & \\
\hline Hematocrit before dialysis (\%) & 32.53 & 6.21 & \multirow{2}{*}{$<0.001$} & 33.89 & 5.91 & \multirow{2}{*}{0.02} \\
\hline Hematocrit after dialysis (\%) & 34.07 & 5.29 & & 34.54 & 4.96 & \\
\hline Serum K before dialysis (meq/L) & 4.85 & 0.69 & \multirow{2}{*}{0.4} & 4.91 & 1.00 & \multirow{2}{*}{0.003} \\
\hline Serum K after dialysis (meq/L) & 3.75 & 0.59 & & 3.60 & 0.49 & \\
\hline
\end{tabular}

with kidney failure often have no problems with the functioning of the RAS system and increase renin levels in response to it. Increases in renin are most commonly found in people with high levels of renin $(13,14)$. However, renin's response to ultrafiltration is already unknown but unmatched in all dialysis patients (5). In our study, the distribution of antihypertensive drugs, including ACEI, was not significantly different between the two groups with and without intradialytic hypertension. Additionally, in our study, the plasma renin activity in the intradialytic hypertension group increased significantly after dialysis compared to pre-dialysis time, but in the control group significantly decreased after dialysis compared to predialysis. The findings of this study are in line with an interventional study by Bazato et al, which has suggested the role of plasma renin activity (4), however, it was contrary to the results of the study by Chou et al (15). An intervention study looked at whether the treatment with ACEI reduces hypertension during dialysis. In this study, the researchers examined six patients with a clear increase in blood pressure during hemodialysis, of which plasma renin activity increased in four of the six patients. Intake of $50 \mathrm{mg}$ of captopril at the start of the hemodialysis session controlled the blood pressure in people whose plasma renin activity increased or not increased. These findings deeply challenged they study by Chou et al (15). They evaluated the hormonal and biochemical status of 30 hemodialysis patients with IDH compared to 30 hemodialysis patients without IDH as the control group before and after dialysis sessions. Antihypertensive drugs stopped for at least 2 weeks. Except for the mean level of arterial blood pressure (MAP), which was higher in the IDH group, there was no significant difference in other parameters between the two groups. In the study of Chou et al (15) plasma renin and norepinephrine levels increased in the control group during the session, but did not change in the IDH group. The lack of plasma renin elevation in IDH patients may question the effectiveness of captopril in blocking the increase in BP during the dialysis session in the study by Bazato et al (4).

In our study, the mean sodium potassium levels did not change after dialysis compared to pre-dialysis in the intradialytic hypertension group. In the control group, the mean sodium $(P=0.6)$ did not change after dialysis, but the mean of potassium decreased significantly. In Chou et al study (15), there was no significant difference between IDH and control group in potassium changes after dialysis and it decreased compared to pre-dialysis in both. In some studies, a positive balance of sodium in extracellular fluid overload and hypertension in dialysis patients is the main mechanism $(16,17)$. Sodium also seems to play a major role in IDH. In hemodialysis patients, when sodium intake is eliminated more during dialysis, the sodium balance is positive. Therefore, in many dialysis patients, a low-salt diet should be recommended (18). In our study, there was no significant difference in the proportion of erythropoietin administered in both groups of patients with and without hypertension during dialysis. The mean dose of erythropoietin in the group suffered hypertension was higher than that of the non-hypertensive group during dialysis, but was not statistically significant.

Some studies have indicated that the prevalence of hypertension in dialysis patients increased by $20 \%-30 \%$ after the extensive use of synthetic erythropoietin. Several potential mechanisms have been mentioned to justify it including increased hematocrit level and resistance to peripheral arteries. Neff et al (19) reported that blood transfusions to a dialysis patient during a 6 to 12 week period for hematocrit of 50 led to increased peripheral vascular resistance, with a significant increase in blood pressure and a decrease in cardiac output. Patients treated with erythropoietin and high hematocrit have similar hemodynamic responses, although dialysis patients may have a change in self-regulated response to increased 
vascular and cardiac outflow $(20,21)$. However, Chou et al (15) do not support this hypothesis. It is important to be cautious before removing the effect of erythropoietin in IDH, since it has been reported that erythropoietin stimulates endothelin synthesis (22).

In our study, the hematocrit level increased in intradialytic hypertension group and control group after dialysis compared to pre-dialysis. Furthermore, the mean of hematocrit before and after dialysis was similar in the two groups. Some studies introduced increasing hematocrit level effective in inducing hypertension during dialysis (19-21).

Mean heart rate decreased significantly in intradialytic hypertension group after first, third and fourth sessions of dialysis, and then increased in the second dialysis session. Mean heart rate decreased significantly in the control group after the first, second, third and fourth dialysis. Therefore, in both groups, the decrease in heart rate was detected. Chou et al (15) also reported the changes in heart rate in both groups, which is consistent with our study.

\section{Conclusion}

In this study, plasma renin activity in the intradialytic hypertension group increased during dialysis and after dialysis compared to pre-dialysis, while in control group it decreased after dialysis compared to pre-dialysis. There was no change in sodium in both groups and potassium in the IHD group, but in the control group, the mean potassium significantly decreased after the dialysis. Hematocrit increased in both groups. Heart rate decreased in both groups. There were no differences in the dose of erythropoietin in the two groups. Therefore, the changes in plasma renin activity in the two groups were the most significant which could be used as a predictive factor.

\section{Limitations of the study}

We conducted a study on a limited proportion of patients. However, larger studies on this concept of hemodialysis are required.

\section{Authors' contribution}

$\mathrm{BH}$ and MRA conducted the research. MRA supervised the study. BH prepared the manuscript. All authors read and signed the final paper.

\section{Conflicts of interest}

The authors declare no conflict of interest.

\section{Ethical considerations}

Ethical issues (including plagiarism, data fabrication, double publication) have been completely observed by the authors.

\section{Funding/Support}

None.

\section{References}

1. Ventura, JE, Sposito M. Volume sensitivity of blood pressure in end-stage renal disease. Nephrol Dial Transplant. 1997;12:485-91.

2. Agarwal R, Flynn J, Pogue V, Rahman M, Reisin, Weir MR. Assessment and management of hypertension in patients on dialysis. J Am Soc Nephrol. 2014;25:1630-46. doi: 10.1681/ASN.2013060601

3. Rocco MV, Yan G, Heyka RJ, Benz R, Cheung AK. Risk factors for hypertension in chronic hemodialysis patients: baseline data from the HEMO study. Am J Nephrol. 2001;21:280-8. doi: 10.1159/000046262.

4. Bazzato G, Coli U, Landini S, Lucatello S, Fracasso A, Morachiello P, et al. Prevention of intra-and postdialytic hypertensive crises by captopril. Contrib Nephrol. 1984;41:292-8.

5. Dorhout MEJ. Rise in blood pressure during hemodialysisultrafiltration: a" paradoxical" phenomenon? Int J Artif Organs. 1996;19:569-70.

6. Inrig JK, Patel UD, Toto RD, Szczech LA. Association of blood pressure increases during hemodialysis with 2-year mortality in incident hemodialysis patients: a secondary analysis of the Dialysis Morbidity and Mortality Wave 2 Study. Am J Kidney Dis. 2009;54:881-90. doi: 10.1053/j. ajkd.2009.05.012.

7. Zager PG, Nikolic J, Brown RH, Campbell MA, Hunt WC, Peterson D, et al. "U" curve association of blood pressure and mortality in hemodialysis patients. Kidney Int. 1998;54:561-9. doi: 10.1046/j.1523-1755.1998.00005.x.

8. Inrig JK, Oddone EZ, Hasselblad V, Gillespie B, Patel UD, Reddan D, et al. Association of intradialytic blood pressure changes with hospitalization and mortality rates in prevalent ESRD patients. Kidney Int. 2007;71:454-61. doi: 10.1038/sj.ki.5002077.

9. Leypoldt JK, Cheung AK, Delmez JA, Gassman JJ, Levin NW, Lewis JA, et al. Relationship between volume status and blood pressure during chronic hemodialysis. Kidney Int. 2002;61:266-75. doi: 10.1046/j.1523-1755.2002.00099.x.

10. Locatelli F, Covic A, Chazot C, Leunissen K, Luño J, Yaqoob M. Optimal composition of the dialysate, with emphasis on its influence on blood pressure. Nephrol Dial Transplant. 2004;19:785-96. doi: 10.1093/ndt/gfh102.

11. Zucchelli P, Santoro A, Zuccala A. Genesis and control of hypertension in hemodialysis patients. Semin Nephrol. 1988;8:163-8.

12. Dolson GM, Ellis KJ, Bernardo MV, Prakash R, Adrogué HJ. Acute decreases in serum potassium augment blood pressure. Am J Kidney Dis. 1995;26:321-6.

13. Travis M, Henrich WL. Henrich. Factors which affect cardiac performance during hemodialysis. Semin Dial. 1989;2:241-5.

14. van der Sande FM, Mulder AW, Hoorntje SJ, Peels KH, van Kuijk WH, Kooman JP, et al. The hemodynamic effect of different ultrafiltration rates in patients with cardiac failure and patients without cardiac failure: Comparison between isolated ultrafiltration and ultrafiltration with dialysis. Clin Nephrol. 1998;50:301-8.

15. Chou KJ, Lee PT, Chen CL, Chiou CW, Hsu CY, Chung HM, et al. Physiological changes during hemodialysis in patients 
with intradialysis hypertension. Kidney Int. 2006;69:18338. doi: $10.1038 /$ sj.ki.5000266.

16. Workgroup KD. K/DOQI clinical practice guidelines for cardiovascular disease in dialysis patients. Am J Kidney Dis. 2005;45:S1-153. doi: 10.1053/j.ajkd.2005.01.019.

17. Locatelli F, Covic A, Chazot C, Leunissen K, Luño J, Yaqoob $\mathrm{M}$, et al. Hypertension and cardiovascular risk assessment in dialysis patients. Nephrol Dial Transplant. 2004;19:105868. doi: 10.1093/ndt/gfh103.

18. Chazot C. Can chronic volume overload be recognized and prevented in hemodialysis patients? Use of a restrictedsalt diet. Semin Dial. 2009;22:482-6. doi: 10.1111/j.1525139X.2009.00642.x.
19. Neff MS, Kim KE, Persoff M, Onesti G, Swartz C. Hemodynamics of uremic anemia. Circulation. 1971;43:876-83.

20. Fellner SK. Intradialytic hypertension: II. Semin Dial. 1993;6:371-3.

21. Nonnast-Daniel B, Schäffer J, Frei U. Hemodynamics in hemodialysis patients treated with recombinant human erythropoietin. Contrib Nephrol. 1989;76:283-9.

22. Bode-Böger SM, Böger RH, Kuhn M, Radermacher J, Frölich JC. Recombinant human erythropoietin enhances vasoconstrictor tone via endothelin-1 and constrictor prostanoids. Kidney Int. 1996;50:1255-61.

Copyright (c) 2018 The Author(s); Published by Nickan Research Institute. This is an open-access article distributed under the terms of the Creative Commons Attribution License (http://creativecommons.org/licenses/by/4.0), which permits unrestricted use, distribution, and reproduction in any medium, provided the original work is properly cited. 